(2) Open Access Full Text Article

\title{
Impact of the COVID-19 Pandemic on Essential Vitreoretinal Care with Three Epicenters in the United States
}

This article was published in the following Dove Press journal: Clinical Ophthalmology

\author{
Sophia El Hamichi' \\ Aaron Gold' \\ Jeffrey Heier ${ }^{2}$ \\ Szilard Kiss ${ }^{3}$ \\ Timothy G Murray (D) \\ 'Miami Ocular Oncology and Retina, \\ Miami, FL, USA; ${ }^{2}$ Ophthalmic Consultants \\ of Boston, Boston, MA, USA; ${ }^{3}$ Weill \\ Cornell Medical College, New York, \\ NY, USA
}

Purpose: To report the impact of COVID-19 on retina practices in three different "hot spot" cities in the United States.

Patients and Methods: The authors assessed data of encounters and intravitreal injections from March 16th to May 8th 2020, at different offices specializing in retina in the United States. All three practices are located in COVID-19 hot spot zones. One practice was in an academic setting, one practice was in a private multispecialty setting, and one practice was a solo private vitreo-retina practice. All practices were focused on emergent/urgent care, and the results were compared to preCOVID-19 weekly averages.

Results: A significant decrease in the number of encounters and injections was revealed in all three centers involved in this review. There was a decrease of $87 \%$ in encounters (156 patients were seen only) and a decrease of 58\% (126 patients) in intravitreal injections in Weill Cornell Medical College in NYC and a decline of 59\% (569 patients) in encounters and a decrease of $64 \%$ (280 patients) of intravitreal injections at the Ophthalmic Consultants of Boston and Tufts University School of Medicine in Boston. The decline at Miami Ocular Oncology \& Retina in Miami was 37\% (1198 patients) in the encounters and 30\% (867 patients) in the injections.

Conclusion: This manuscript documents a specific example illustrating that COVID-19 has led to a significant decrease in specialized health services. The degree of infection and mortality rate at each hot spot had a direct impact on the practice volume; however, the type of practice setting also played a role.

Keywords: COVID-19, pandemic, retina, ophthalmology, medical practice

\section{Introduction}

In just a few months, the COVID-19 pandemic has reshaped the modern world in unprecedented ways. It has raised fear, anxiety, and uncertainty and it has abruptly changed our way of life. On January 20th, 2020, the Centers for Disease Control and Prevention (CDC) confirmed the first case of COVID-19 in the United States, in the state of Washington. ${ }^{1}$ The first death from coronavirus in the US was then reported in the state of Washington on February 29th. ${ }^{1}$ Public activities began to cease, businesses began to close, and restrictions were expanded, with stay-at-home orders issued on March 20th in New York, March 22nd in Massachusetts, and March 24th in Florida. On March 21st, the ASRS issued recommended measures for seeing patients and defined the urgent and emergent case guidelines in accordance with guidelines from many state health departments, the AAO, and the US
Correspondence: Timothy G Murray Miami Ocular Oncology and Retina, Miami, FL 33143, USA

Email tmurray@murraymd.com 
Surgeon general's warning. ${ }^{2,3}$ Broadly, the universal strategy to combat this pandemic has been to flatten the infection rate curve in order to prevent medical facilities across the world from getting overwhelmed with severe cases. This strategy has generally been implemented in the form of social isolation. Most governments issued stay-at-home orders to their citizens and many businesses that were deemed "non-essential" were temporarily closed. The healthcare industry is essential; however, medical priority has turned toward patients affected by the virus. Medical practices in virtually all specialties not directly related to the novel coronavirus have experienced drastic changes. The number of patient encounters and the number of medical procedures have dramatically fallen. In ophthalmology, office visits were broadly reduced to urgent and emergent cases only, with most routine visits being delayed and elective surgeries postponed. ${ }^{2,3}$ The purpose of this manuscript is to describe the pandemic's effect on three different retina practice modalities in three different hot spots in the United States. This report will also highlight the importance of preparedness for early care in the event of an unexpected disaster. ${ }^{4}$

\section{Patients and Methods}

This report reviews the data of patients seen from March 16th to May 8th, 2020, as national awareness rose against the virus. These patients were seen in three different locations: The vitreoretinal service of Ophthalmic Consultants of Boston (OCB) in Boston, Massachusetts; The Department of Ophthalmology, Weill Cornell Medical College, New York-Presbyterian Hospital in New York, New York; and Miami Ocular Oncology and Retina (MOOR) in Miami, Florida. The data analyzed in this manuscript represents patient encounters from a single retina specialist at each center.

\section{Patients Eligibility and Exclusion Criteria}

The authors included urgent and emergent patients, defined by the American Society of Retina Surgeons (ASRS) and the American Academy of Ophthalmology (AAO) guidelines. ${ }^{2,3}$

Patients that benefited from virtual visits or phone call encounters, urgent and emergent surgery cases, pediatric patients that required examination under anesthesia, and premature infants that were examined in the neonatal intensive care unit were not included in this review. Patients included in this review were seen in the clinic after implementing the following guidelines: $:^{2,3,5-7}$ Prior to the visit, patients were educated on adequate measures to protect themselves and others via email and/or social media. During appointment confirmation calls, all patients were reminded to wear a mask to the office and were asked about any COVID-19 related symptoms or suspicious contacts. If they exhibited any COVID-19 related symptoms, patients were asked to self-isolate, and their appointment was rescheduled to at least two weeks into the future or they were referred to hospitals equipped to manage COVID-positive patients. For patients without symptoms, appointment times were spaced out between patients. Additionally, patients who wished to wait in their car after checking in were given the opportunity to do so. Furthermore, patients' companionship was limited.

Once in the office, pertinent COVID-19 symptoms were rechecked with all patients at the front desk, as well as if there was any contact with infected or suspected infected individuals. If there was any evidence of one of these previous criteria, the patient was immediately sent home and it was recommended that they contact their primary care physician. Social distancing rules were applied, particularly in the waiting area. Wearing a mask for both patients and personnel was required. The rooms, ophthalmic equipment, and contact surfaces were vigorously cleaned with disinfectant between patients.

\section{Data Collection and Assessment of Bias}

The collection of data was collected and reviewed from the three locations accordingly. The analysis was then focused on each center's performance before the pandemic and the performance evolution throughout the initial 8 weeks of the pandemic. The limitations of this study are: the small number of locations sampled; the minor disparity of the retina practices included in the review, as two of the practices are in a private setting (MOOR and OCB) and one is in an academic setting (Weill Cornell); and the geographic location, as this study only reports the experience of 3 retina practices in the USA. However, the goal of this review is not a comparison of the impact of the pandemic throughout multiple regions; rather, it is meant to be a historical snapshot on how the pandemic has affected these different retina practices in different locations. The encounter and treatment data during the pandemic have been compared to the pre-COVID-19 weekly averages.

This study is approved by Larkin Hospital Institutional Review Board, Miami, Florida, USA (affiliated with MOOR). The patient's consent waiver is assigned for retrospective 
studies. The patient's data confidentiality is respected, and this study is compliant with the Declaration of Helsinki.

\section{Results}

For a period of 8 weeks, from March 16th to May 8th, 2020, there was a significant drop in patients' visits and intravitreal injections. In OCB, there were 569 encounters and 280 injections, with an average of 71 encounters per week and 35 injections per week. This represents a drop of 59\% for encounters and $64 \%$ for injections, as the weekly average of encounters prior to COVID-19 crisis was 156 encounters per week and 98 injections per week. The highest drop was registered the week of April 6th with a decline of $75 \%$ in encounters (43 encounters) and a decline of $76 \%$ in injections (23 injections performed). (Figure 1 and Table 1)

In the Weill Cornell clinic, during the same 8 weeks, there were 156 in encounters and 126 in injections. The average of encounters was 19 per week, which was $87 \%$ lower than the average seen before the pandemic, 152 encounters per week. Similarly, the average number of injections per week declined by $58 \%$ with a new average of 15 injections post-COVID-19 compared to an average of 38 injections per week pre-COVID-19. The steepest fall in these numbers was registered the week of March $30^{\text {th }}$ with a decline of $100 \%$ in both encounters and injections (0 patients). (Figure 1 and Table 1)

In MOOR, there was a total of 1198 patient encounters and a total of 867 intravitreal injections with an average of 149 encounters per week and 108 injections per week. Following the pandemic surge, there was a weekly average drop of $39 \%$ in encounters compared to the pre-COVID-19 average number set at 240 encounters per week. The highest drop of 53\% occurred the week of March 23rd with 112 encounters. Similarly, there was a decrease of $30 \%$ in the weekly average of intravitreal injections, compared to the pre-COVID-19 average number set at 156 injections per week (Figure 1). The highest decrease of intravitreal injections was also seen the week of March 23rd, with a drop of $46 \%$ with 84 injections (Figure 1 and Table 1).

When comparing the percentages of drops in encounters and injections in these 3 locations, it is clear that these hot spot zones were affected differently. Weill Cornell was the most affected, as the drop throughout this period was between $76 \%$ and $100 \%$ in patient encounters; to a lesser

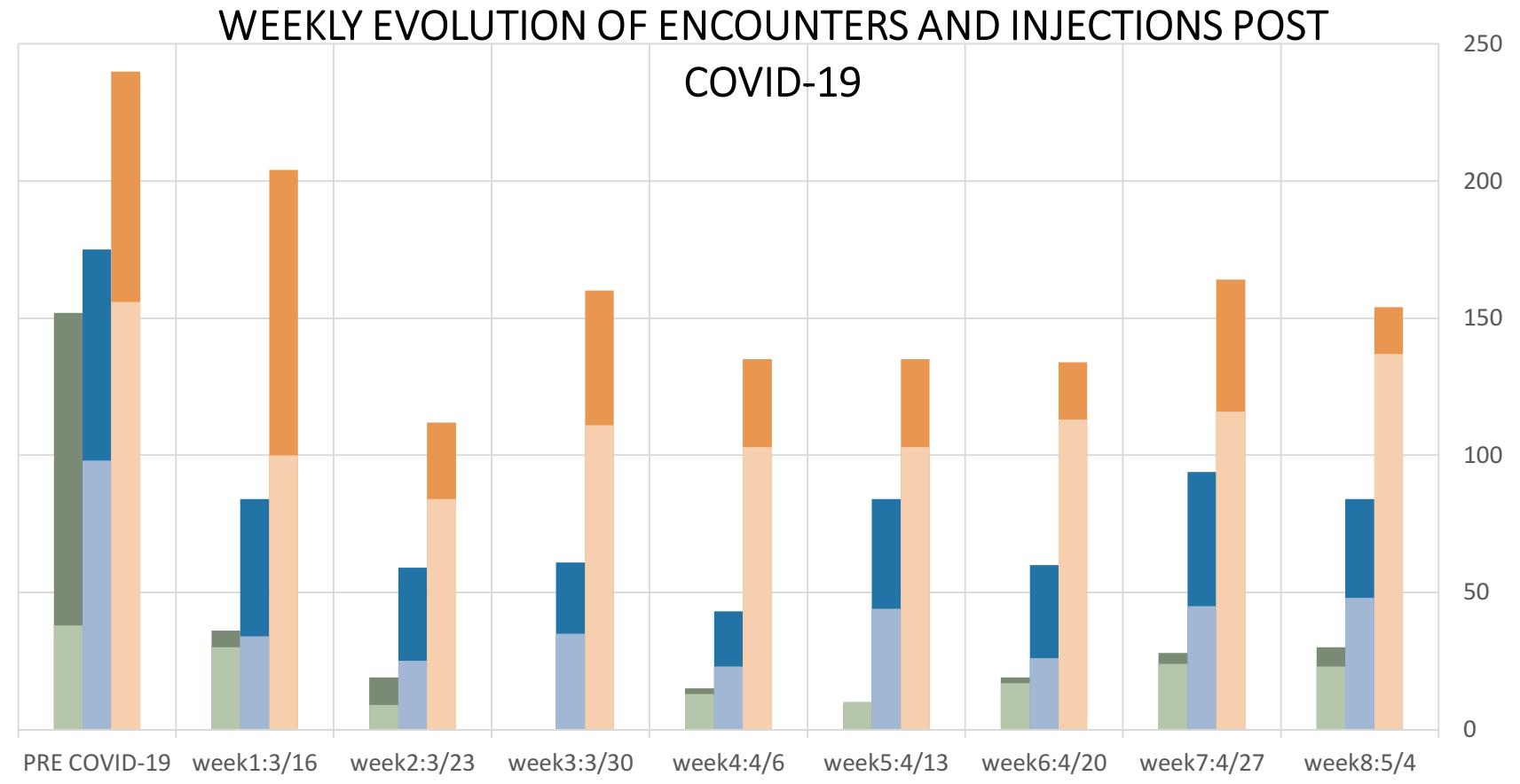

MOOR ENCOUNTERS

MOOR INJECTIONS

Figure I Weekly evolution of encounters and intravitreal injections during the initial 8 weeks of COVID-19 pandemic. 
Table I Weekly Percentage of Decrease in Encounters and Intravitreal Injections in the 3 Retina Practices During the Initial 8 Weeks of COVID-19 Pandemic

\begin{tabular}{|l|l|l|l|l|l|l|}
\hline & Encounters & Encounters & Encounters & Injections & Injections & Injections \\
\cline { 2 - 6 } & Weill Cornell & OCB & MOOR & Weill Cornell & OCB & MOOR \\
\hline Pre COVID-19 & 152 & 156 & $\mathbf{2 4 0}$ & $\mathbf{3 8}$ & $\mathbf{9 8}$ & $\mathbf{1 5 6}$ \\
\hline Week I: $3 / 16$ & $36(76 \%)$ & $84(52 \%)$ & $204(15 \%)$ & $30(21 \%)$ & $34(65 \%)$ & $100(35 \%)$ \\
Week 2: $3 / 23$ & $19(87 \%)$ & $59(66 \%)$ & $112(53 \%)$ & $9(76 \%)$ & $25(74 \%)$ & $84(46 \%)$ \\
Week 3: $3 / 30$ & $0(100 \%)$ & $61(65 \%)$ & $160(33 \%)$ & $0(100 \%)$ & $35(64 \%)$ & $111(28 \%)$ \\
Week 4: $4 / 6$ & $15(90 \%)$ & $43(75 \%)$ & $135(43 \%)$ & $13(65 \%)$ & $23(76 \%)$ & $103(33 \%)$ \\
Week 5: $4 / 13$ & $9(94 \%)$ & $84(52 \%)$ & $135(43 \%)$ & $10(73 \%)$ & $44(55 \%)$ & $103(33 \%)$ \\
Week 6: $4 / 20$ & $19(87 \%)$ & $60(65 \%)$ & $134(44 \%)$ & $17(55 \%)$ & $26(73 \%)$ & $113(27 \%)$ \\
Week 7: $4 / 27$ & $28(81 \%)$ & $94(46 \%)$ & $164(31 \%)$ & $14(36 \%)$ & $45(54 \%)$ & $116(25 \%)$ \\
Week 8: $5 / 4$ & $30(80 \%)$ & $84(52 \%)$ & $154(35 \%)$ & $23(39 \%)$ & $48(51 \%)$ & $137(12 \%)$ \\
8 weeks average percentage decrease & $87 \%$ & $59 \%$ & $37 \%$ & $58 \%$ & $64 \%$ & $30 \%$ \\
\hline
\end{tabular}

Note: * patients present (percentage decrease).

extent, the impact on the percentage of injections varied between $21 \%$ and $100 \%$. The Boston location in this study, OCB, was the second most affected, with a decline of encounters between $46 \%$ and $75 \%$, and a decline in injections between $51 \%$ and $76 \%$. MOOR in Miami was the least impacted of the three centers, with a decline of patient encounters between $15 \%$ and $53 \%$, and in injections between $12 \%$ and $46 \%$. (Figure 1 and Table 1)

\section{Discussion}

The first confirmed infection in Massachusetts was registered in Boston on February 1st and the first confirmed infected cases in New York state and Florida were both announced on March 1st. As of May 9th, the death tolls from COVID-19 had reached 26,683 in New York State; 4898 in Massachusetts, and 1631 in Florida. ${ }^{8}$

There was a significant impact of the pandemic on ophthalmology practices in general, and retina practices specifically; however, the impact on retina practices was variable given the critical importance of maintaining treatment with anti-VEGF injections in diseases such as neovascular age-related macular degeneration (AMD), diabetic macular edema, retinal venous occlusive disease, diseases which could result in irreversible vision loss without such treatment. The degree of impact was felt differently in different hot spot zones. For instance, the impact in NYC was significantly higher than the other areas; it reached $100 \%$ with no retina cases seen in the week of March 30th, 2020. Following that week, numbers in NY continued to be low. This may be explained by several factors: Firstly, the death toll and number of cases were significantly higher in New York. Secondly, in New York, and to a lesser extent in Boston, some of the ophthalmic healthcare providers were redeployed to assist with COVID-19 infection cases in emergency rooms and intensive care units. This was especially true for academic centers, such as Cornell. Most academic settings closed their clinics to non-COVID-19-related specialties. As a result, the ophthalmic workforce was lower. In hot spot areas, many private ophthalmology practices were also widely shut down, and the few that stayed open were very restricted, as patients with COVID-19 were prioritized in most hospitals. In this way, practice setting played a major role in patient volume during the pandemic. Patients may also have been hesitant to come to visits in areas with such high profile disease rates such as NY and Boston.

Furthermore, the pandemic impact on jobs has widely affected these regions and many ophthalmology practices have had to lay off or furlough their employees. The approach affecting the staffing was different, depending on each ophthalmology practice and its individual characteristics.

South Florida deals with natural disasters more frequently than the rest of the country due to frequent threats of hurricanes annually. ${ }^{4}$ Although the COVID-19 pandemic is a vastly different disaster when compared to a hurricane, state-wide disaster readiness may have aided practice management decisions regarding this novel disaster. The Miami practice's implementation of strict measures protecting its staff and the patients allowed patients to gain confidence in being safe while being seen. Access to appropriate PPE, appropriate patient screening, and a commitment to our patients and our staff helped prevent 
the need to furlough employees in Miami. Furthermore, despite the risk of infection and state-wide stay-at-home orders, many patients understood the need to visit the office, as their risk of losing sight or having a tumorrelated complication outweighed their fear of contracting COVID-19. This difference in patients' perspective is probably additionally impacted by the fact that there was less impact from the virus in Florida compared to the other hot spot zones, with less infected cases and deaths. Although the threat of infection was the ultimate concern for patients, other contributing exacerbated uncertainty surrounding the virus. One of the concerns that the pandemic revealed was the virus testing availability. ${ }^{1}$ This concern was felt by both healthcare workers and the patients. Fortunately, virus testing possibilities are currently becoming increasingly available. This is particularly beneficial for the medical personal and patients undergoing surgeries. It also allows a wider scale of testing and thus infection prevention, as the number of asymptomatic cases spreading the virus is thought to be considerable. ${ }^{9,10}$ The pandemic has also disrupted manufacturing. Fortunately, there have not been significant shortages in ophthalmic medications necessary for patient care. Shortages were felt in masks and other PPE, and it was preferable that patients bring their own masks, if possible. Similarly, no shortages of prescribed drugs have been reported from the patients thus far. It was crucial to ensure the continuation of medical therapies production so that they were made available for our patients in a timely manner; this also applied to PPE and the mask shortage situation that is starting to be resolved.

Another issue the pandemic has raised is the subject of remote care and telemedicine. Telemedicine is a practice that has emerged in recent years and COVID-19 pandemic has brought this technology to the spotlight. Numerous studies have demonstrated that telemedicine is as safe and accurate as face to face encounters. ${ }^{11-13}$ Nevertheless, the limits of telemedicine are undeniable when it comes to certain diseases as imaging and further examination steps are required. However, this can be resolved by developing and innovating technology that will allow imaging from a distance, ${ }^{13}$ and possibly link them to an approved system of artificial intelligence to minimize errors and ensure more accuracy. ${ }^{14-19}$ Telemedicine allows remote care, avoids unnecessary patient visits, but is also cost-effective when it comes to socioeconomics and geographic barriers for patients. ${ }^{12,20}$

Nevertheless, in most retina practices, telemedicine in its current form did not prove to be helpful enough in terms of taking care of complex patients, as the majority of them required imaging and treatment. Treatment delay is directly related to vision loss in patients with chronic vascular activity. Furthermore, the elderly population did not feel comfortable to switch to telemedicine, which may require learning and adjusting skills. Perhaps in the future, telemedicine for retinal patients, especially those receiving injections, will be more applicable when advancements in home monitoring, such as home OCT (now in development) are available.

\section{Conclusion}

Although this manuscript is not a comprehensive evaluation of the effects of the pandemic on all of ophthalmology, these results show that the three centers that were evaluated were significantly affected by the global COVID-19 outbreak. Living with the threat of COVID19 has currently become the new normal. However, patients that require ongoing treatment are at risk of severe vision loss if treatment is delayed for a sustained period of time. To that end, a preparedness plan to aid in the management of specialized health care is warranted. While there is hope that a vaccine or an efficient therapy will soon be available, a proper adjustment with adopting strict safety measures is suggested, and a better understanding of the virus' behavior will help improve our safety protocols. Although there is no clear perfect approach yet, we believe that implementing the safety measures discussed in this manuscript, such as universal mask-wearing, patient communication, and aggressive office disinfecting will aid in maintaining patients' vision and quality of life.

\section{Disclosure}

Aaron Gold reports personal fees from Regeneron, outside the submitted work. Jeffrey Heier reports personal fees from 4DMT, Adverum, Aerie, Aerpio, Aldeyra, Allegro, Alzheon, Annexon, Apellis, Asclepix, Aviceda, BeaverVisitec, Eloxx, Galimedix, Genentech, Gyroscope, iRenix, jCyte, Kala, Kanghong, NGM, Notal Vision, Novartis, Ocugenix, Oculis, Ocunexus, Ocular Therapeutix, Palatin, Pfizer, Regeneron, Regenxbio, Santen, Scifluor, Shire, Stealth, Tyrogenex, Vinci, Voyant, grants from Aerie, Aerpio, Apellis, Genentech, Graybug, Gyroscope, Hemera, Janssen R\&D, KalVista, Kanghong, Novartis, Ophthotech, Optovue, Regeneron, Regenxbio, Stealth, outside the submitted work. Szilard Kiss reports Consultant - Adverum, Alcon, Allergan, BioMarin, Novartis, Optos, Genentech/ Roche, Regeneron; Research Funding - Allergan, 
Novartis, Optos, Genentech/Roche, Regeneron; Equity Adverum, RegenXBio, Fortress Bio; Intellectual Property - Gene Therapy for AMD, T-Cells for CMV Retinitis. The authors report no other potential conflicts of interest for this work.

\section{References}

1. Centers for Disease Control and Prevention. CDC newsroom releases. Available from: https://www.cdc.gov/media. Accessed April 17, 2020.

2. American Academy of Ophthalmology (AAO). Important coronavirus updates for ophthalmologists, Available from: https://www.aao.org/head line/alert-important-coronavirus-context. Accessed April 17, 2020.

3. American Society of Retina Specialsts (ASRS). COVID-19 updates and resources. Available from: https://www.asrs.org/practice/asrs-memberalert-regarding-covid-19-pandemic. Accessed April 17, 2020.

4. El Hamichi S, Gold A, Kon Graversen V, et al. Disaster preparedness impact in a complex ophthalmology practice: a review of patients receiving intravitreal injections. Clin Ophthalmol. 2019;11(13):19992002. doi:10.2147/OPTH.S224454

5. The L. COVID-19 protecting health-care workers. Lancet. 2020;395 (10228):922. doi:10.1016/S0140-6736(20)30644-9

6. Seittzman GD, Doan T. No time for tears. Ophthalmology. 2020;127 (7):980-981. doi:10.1016/j.ophtha.2020

7. Olivia Li JP, Shantha J, Wong T, et al. Preparedness among ophthalmologists: during and beyond the COVID-19 pandemic. Ophthalmology. 2020;127:569-572. doi:10.1016/j.ophtha.2020.03.037

8. Institute of health metrics and evaluation. COVID-19 projections. Available from: https://covid19.healthdata.org/united-states-of-amer ica. Accessed June 8, 2020.

9. Pan X, Chen D, Xia Y, et al. Asymptomatic cases in a family cluster with SARS-CoV-2 infection. Lancet Infect Dis. 2020;20:410-411. doi:10.1016/S1473-3099(20)30114-6

10. Luo SH, Liu W, Liu ZJ, et al. A confirmed asymptomatic carrier of 2019 novel coronavirus (SARS-CoV-2). Chin Med J (Engl). 2020;133(9):1123-1125. doi:10.1097/CM9.0000000000000798
11. Hailey D, Roine R, Ohinmaa A. Systematic review of evidence for the benefits of telemedicine. J Telemed Telecare. 2002;8(1_suppl):17. doi:10.1258/1357633021937604

12. Sreelatha OK, Ramesh SV. Teleophthalmology: improving patient outcomes? Clin Ophthalmol. 2016;10:285-295. doi:10.2147/OPTH. S80487

13. Tan IJ, Dobson LP, Bartnik S, Muir J, Turner AW. Real-time teleophthalmology versus face-to-face consultation: a systematic review. J Telemed Telecare. 2017;23(7):629-638. doi:10.1177/1357633X16660640

14. DeBuc DC. The role of retinal imaging and portable screening devices in tele-ophthalmology applications for diabetic retinopathy management. Curr Diabetes Rep. 2016;16(12):132. doi:10.1007/ s11892-016-0827-2

15. Keskinbora K, Güven F. Artificial intelligence and ophthalmology. Turk J Ophthalmol. 2020;50(1):37-43. doi:10.4274/tjo.galenos.2020.78989

16. Abràmoff MD, Lavin PT, Birch M, Shah N, Folk JC. Pivotal trial of an autonomous AI-based diagnostic system for detection of diabetic retinopathy in primary care offices. Npj Digital Medicine. 2018;1:39. doi:10.1038/s41746-018-0040-6

17. Grassmann F, Mengelkamp J, Brandl C, et al. A deep learning algorithm for prediction of age-related eye disease study severity scale for age-related macular degeneration from color fundus photography. Ophthalmology. 2018;125:1410-1420. doi:10.1016/j.ophtha. 2018.02.037

18. Chakrabarty L, Joshi GD, Chakravarty A, Raman GV, Krishnadas SR, Sivaswamy J. Automated detection of glaucoma from topographic features of the optic nerve head in color fundus photographs. J Glaucoma. 2016;25:590-597. doi:10.1097/IJG.0000000000000354

19. Redd TK, Campbell JP, Brown JM, et al. Imaging and Informatics in Retinopathy of Prematurity (i-ROP) Research Consortium. Evaluation of a deep learning image assessment system for detecting severe retinopathy of prematurity. Br J Ophthalmol. 2018;313156.

20. Avidor D, Loewenstein A, Waisbourd M, Nutman A. Cost-effectiveness of diabetic retinopathy screening programs using telemedicine: a systematic review. Cost Eff Resour Alloc. 2020;18:16. doi:10.1186/ s12962-020-00211-1
Clinical Ophthalmology

\section{Publish your work in this journal}

Clinical Ophthalmology is an international, peer-reviewed journal covering all subspecialties within ophthalmology. Key topics include: Optometry; Visual science; Pharmacology and drug therapy in eye diseases; Basic Sciences; Primary and Secondary eye care; Patient Safety and Quality of Care Improvements. This journal is indexed on PubMed

\section{Dovepress}

Central and CAS, and is the official journal of The Society of Clinical Ophthalmology (SCO). The manuscript management system is completely online and includes a very quick and fair peer-review system, which is all easy to use. Visit http://www.dovepress.com/ testimonials.php to read real quotes from published authors. 